\title{
LEIBNIZ Y LA FILOSOFÍA DE LA RELIGIÓN EN NISHIDA KITARÔ
}

Agustín Jacinto Z.* jacintoz@colmich.edu.mx

RESUMO Este trabalho é uma análise da forma pela qual Nishida Kitarô (1870-1945), no processo de construção de sua própria filosofia da religião, entra em diálogo com o pensamento de Leibniz relativo à Harmonia Pré-Estabelecida. Embora a filosofia da religião seja um tema importante, e Nishida retome Leibniz em alguns pontos em sua carreira textual, existem relativamente poucos estudos que abordem a relação entre esses dois pensadores. Estudo a abordagem de Nishida sob três categorias. A primeira seção refere-se a dez principais aspectos do mundo de harmonia preestabelecida. A segunda busca demonstrar o modo pelo qual esse mundo se torna um guia para uma filosofia da religião, colocando a ênfase em três aspectos caracteristicamente cristãos: um mundo criado, caído e trinitário. A terceira seção inclui alguns elementos, tais como a entrada na religião, a metanoia, o satori e uma comparação entre o Ágape cristão e o Maha-Kruna budista. Estes são temas sobre os quais Nishida elabora em sua obra-prima de 1945, A Lógica de Basho e uma Visão Religiosa do Mundo.

Palavras-chave Nishida, Leibniz, harmonia preestabelecida, filosofia da religião.

ABSTRACT This paper is an analysis of the manner Nishida Kitarô (1870-1945), in the process of construction of his own philosophy of religion,

* Colegio de Michoacán, Zamora, México. Artigo recebido em 11/01/2015 e aprovado em 02/09/2015.

KRITERION, Belo Horizonte, nº 133, Abr./2016, p. 207-232 
enters into dialogue with Leibniz's thought concerning Pre-established Harmony. Although the philosophy of religion is an important theme and Nishida goes back to Leibniz at some points in his textual career, there are relatively few studies that touch on the relationship between these two thinkers. I study Nishida's approach under three headings. The first section concerns ten main aspects of the world of pre-established harmony. The second tries to show the manner in which such a world becomes a guide towards a philosophy of religion, placing the emphasis on three characteristically Christian aspects: a created, fallen, and Trinitarian world. The third section includes some elements such as the entrance into religion, metanoia, satori and a comparison between Christian agape and Buddhist maha-kruna. These are themes on which Nishida elaborates in his 1945 masterpiece, The Logic of Basho and a Religious Worldview.

Keywords Nishida, Leibniz, pre-established harmony, philosophy of religion.

\section{Introducción}

En la actualidad el estudio de las relaciones entre Leibniz y Nishida se centran principalmente en la teoría de la información ${ }^{1}$ la influencia de la monadología, ${ }^{2}$ en especial la mónada creativa, ${ }^{3}$ el si-mismo, ${ }^{4}$ los sistemas complejos, ${ }^{5}$ la ecología. ${ }^{6}$

Sin embargo, en el estudio de la filosofía de la religión de Nishida resulta también necesario examinar el diálogo de Nishida con Leibniz. A través de la reflexión contrastante con el pensamiento de Leibniz, Nishida logra -en el periodo tardío de su pensamiento- clarificar algunos puntos esenciales para la construcción de una filosofía de la religión desde su propio punto de vista. Para ello se apoya principalmente en la "Monadología” (Diario, 28ii1905), el "Discurso sobre la metafísica", los "Nuevos ensayos sobre el entendimiento humano" (Diario, enero 9 de 1907), el "De arte combinatoria” que él denomina

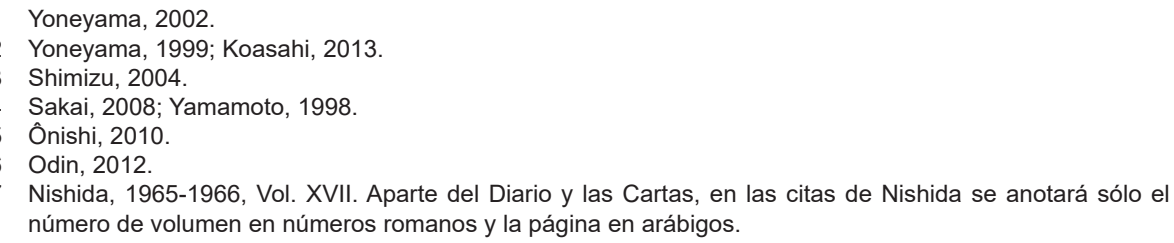


Kombinatorik, la "Correspondencia con Arnauld" (Carta \#2409, enero 16 de 1918, a Tanabe Hajime), y dispone también de las "Oeuvres philosophiques de Leibniz". ${ }^{8}$ Además, para su interpretación de Leibniz se apoya en Louis Couturat, ${ }^{9}$ Bertrand Russell ${ }^{10}$ y Gabriel Tarde.

Nishida recibió la influencia de la "mónada abierta" de Tarde, quien como dice Éric Alliéz propuso "mónadas abiertas que recíprocamente se interpenetran en lugar de ser exteriores unas a otras" y cuya interpretación de Leibniz impactó también a Deleuze, Guattari, Simmel y Foucault. ${ }^{11}$

Por razones de espacio no mencionaré otros textos en que Nishida hace referencia a Leibniz y tomaré como base su escrito "Hacia la filosofía de la religión tomando como guía la Armonía Preestablecida", ${ }^{12}$ que es uno de los textos nishidianos menos estudiados por los especialistas, a pesar de que en él podemos ver un antecedente de "Lógica del basho y cosmovisión religiosa" (XI: 371-464), la obra maestra del periodo nishidiano tardío. Encontramos muy pocos escritos que examinen la filosofía nishidiana de la religión en su confrontación con la propuesta de Leibniz. ${ }^{13}$

El escrito de Nishida que aquí nos ocupa puede ser estudiado en dos partes: en una primera parte veremos qué es la armonía preestablecida y cómo es su mundo; y en una segunda, de qué manera esa armonía preestablecida nos lleva a una filosofía de la religión.

\section{I}

\section{Caracterización del mundo de la armonía preestablecida}

Vamos a presentarvarias perspectivas del mundo de la armonía preestablecida que menciona Nishida. En general se distinguen tres periodos en la recepción del concepto de la armonía preestablecida, ${ }^{14}$ pero Nishida retoma una formulación de Leibniz que implica:

8 Janet, 1900; Nishida, Carta \#2410, 23 enero de 1918, a Tanabe Hajime.

9 Couturat, 1901.

10 Russell, 1900.

11 Tarde, 1999, pp. 31-32.

12 Este texto fue publicado en mayo-junio de 1944 en la revista Shisô. Aquí vamos a utilizar el texto contenido en XI: 114-146.

13 Ônishi, 2012; Kurusu, 2005, como Apéndice incluye la traducción del texto que aquí comentamos "Auf dem Weg zur Religionsphilosophie- geleitet von der prästabilierten Harmonie” (1944), pp. 111-136. Sólo he tenido acceso al índice.

14 Masuyama, 2013. 


\subsection{Un mundo activo}

Nishida nos dice que el mundo de que habla es un mundo activo en el que se da siempre la mutua oposición entre individuos (es decir, entre los elementos de ese mundo) y esa mutua oposición es también una acción (XI: 114). En un mundo activo resulta inadecuada e insuficiente la definición aristotélica que nos dice que el individuo es sujeto que no deviene predicado. La razón es que el mundo activo es visto como un topos (basho) en el que se da aquella mutua oposición: es un espacio histórico y, al mismo tiempo, un "mundo del presente absoluto" (XI: 115).

Leibniz no coloca en el mundo histórico el origen de la mutua oposición entre los individuos. Más bien, dice que en el mundo cada individuo es enteramente real ya que "ni se origina ni muere y sólo es creado y destruido por Dios". Por esto, la multiplicidad de los individuos también es real, y el origen de la mutua relación entre éstos también está solamente en Dios. De esta manera, aunque la mutua oposición entre individuos se da en el mundo, el origen del mundo, de los individuos y de la mutua oposición entre ellos está en Dios. Por tener un solo referente y un solo origen, puede pensarse la armonía preestablecida.

Nishida dice que aunque en filosofía se ha pensado que el concepto de individuo encierra contradicción, "todavía no se ha construido una nueva lógica a partir de allí” (XI: 115). Por esto, igual que para Leibniz, no es suficiente con que el yo se autoperciba: la constitución misma del individuo se da por su oposición frente a todo otro individuo: el evento mismo del surgimiento del individuo implica contradicción, implica que sólo pueden existir los individuos en su mutua oposición. Mientras que en la lógica aristotélica "el individuo se sostiene a sí mismo" como substancia individual, este no es el caso en la lógica tópica de Nishida, en la que al ser insubstanciales, todos los individuos se constituyen mediante su mutua oposición: se constituyen en "la autoidentidad de contradictorios" que es una relación de existencia mediante su mutua interdependencia.

Nishida explica la autoidentidad de contradictorios de la siguiente manera: la autoidentidad de contradictorios implica que la "negación absoluta $\equiv$ afirmación absoluta" es "contradicción三identidad". Para que no pueda quedar el malentendido de que se refiere a categorías aristotélicas, Nishida dice: "a esto le podemos llamar autodeterminación de la Nada absoluta" (XI: 115). La autoidentidad de contradictorios es también el caso en la relación entre el todo y el individuo, entre lo múltiple y lo uno, y otros conceptos que en la lógica aristotélica no pueden ser idénticos. En el mundo histórico activo que tiene carácter de autoidentidad entre espacio y 
tiempo, puede también expresarse la autoidentidad de contradictorios como "autodeterminación del presente absoluto" (XI: 115-116).

Así el mundo de Leibniz puede ser visto como un mundo del presente absoluto en el que "Dios, como autoidentidad de lo absolutamente contradictorio, debe ser presente absoluto", y el mundo puede ser visto como "un mundo de la creación y, al mismo tiempo, un mundo de la originación y desaparición” (XI: 116). Es básicamente un mundo activo.

\subsection{Un mundo autoexpresivo}

En términos de Leibniz habría que decir que "Dios en cuanto presente absoluto se expresa a sí mismo enteramente" y que el individuo como "espejo de Dios", ${ }^{15}$ "expresa al mundo entero de diversas maneras". ${ }^{16}$ La mónada que se expresa a sí misma, en tanto que lo expresante es lo expresado, es mutua relación con otra(s) mónada(s): es un punto de la perspectiva del mundo e incluye "dentro de sí a la expresión infinita" (XI: 116). Una mónada expresa dentro de sí a las otra(s) mónada(s): es activa. Leibniz percibió que "lo que más claramente expresa al mundo es lo más activo" (XI: 116-117). Pero Leibniz se coloca en la tradición de los griegos entre quienes la forma es lo activo: "pensó el mundo de la fuerza desde el punto de vista de la forma substancial" y desde este punto de vista la forma es activa y la materia es pasiva. Es decir, preso en la lógica griega "Leibniz no llegó a percibir el principio lógico de la autoidentidad de lo contradictorio" (XI: 117).

Para Nishida, el principio lógico de la constructividad del mundo histórico no radica en que la forma construya a la materia sino en "las relaciones de autoidentidad de lo contradictorio". Dentro de estas relaciones -que incluyen la mutualidad entre lo múltiple y lo uno-, cada individuo refleja "a todo el mundo dentro de sí mismo [y esto] es ir de la multiplicidad individual a la unidad total", es expresar a todo el mundo, es formar el mundo, es trabajar "en cuanto autodeterminación del mundo entero". Al mismo tiempo, ir "de la unidad total a la multiplicidad individual: es construir, es actuar, [porque] actuar mediante el hecho de que el individuo exprese en sí mismo al mundo entero debe significar que el individuo [...] es expresado, es reflejado dentro de un mundo así" (XI: 117-118). 


\subsection{Un mundo autorreflexivo}

Tanto en el caso del todo como en el caso del individuo, ser expresado es ser reflejado: el individuo "es expresado en este mundo y, al mismo tiempo, se expresa a sí mismo", de tal manera que todo "lo que existe es algo que es reflejado y que refleja". Este mundo de la mutua expresión y la mutua reflexión, del mutuo expresar y ser expresado, del mutuo reflejar y ser reflejado, es un mundo activo en el que todo individuo es igualmente activo. Pero ese mutuo reflejo no es reflexión de la substancia del mundo o del individuo: "el mundo es reflejado autonegativamente dentro de sí mismo" y allí se originan las cosas, al mismo tiempo, cuando el mundo "autoafirmativamente se refleja a sí mismo dentro de sí, las cosas actúan". En este sentido, "el mundo comienza a partir de que se refleja a sí mismo" (XI: 118). Desde este punto de vista debe pensarse "la relación entre forma y materia", porque "ser reflejado es reflejar, y reflejar es ser reflejado". Esto implica que en vez de pensar que la forma es activa y la materia pasiva se llegue a pensar que "la forma se determina a sí misma con carácter de autoidentidad de lo contradictorio: forma y materia" (XI: 118-119).

\subsection{Un mundo de la Nada absoluta}

Para poder pensar la infundamentación del mundo de la realidad absoluta que va de lo hecho a lo activo, es necesario hacerlo mediante una lógica concreta de las relaciones de la actividad, de la expresión y del reflejo. Nishida busca una lógica dinámica concreta. En este sentido el Dios de Spinoza debería ser pensado como "una forma infinita que se determina a sí misma, [es decir,] una forma sin forma". En términos de Nishida: "Dios es Nada absoluta", o bien, "Dios es un espejo eterno, podemos decir que es "un gran espejo redondo" (X: 119). ${ }^{17}$ Frente a un Dios que es sujeto absoluto -como en Spinoza, ${ }^{18}$ para quien finalmente Dios se convirtió en "un sustrato estático abstracto"-, se puede pensar que todo cuerpo material "es un modo que expresa a la esencia de Dios en tanto que extensa". De allí surge la idea de una "natura naturans $\equiv$ natura naturata" (XI: 119-120) como identidad de los modos: "La forma que se determina a sí misma en cuanto natura naturans, y la forma que es determinada, en cuanto natura naturata, son modos" (XI: 122) 


\subsection{Un mundo de la armonía preestablecida}

En este acercamiento a Leibniz, Nishida relaciona la expresión, lo reflejo y la Nada absoluta, de manera que de ahí resulta "un mundo [en el que] un futuro eterno es reflejado en el pasado eterno", por lo que ese mundo es omni-incluyente. Cuando se piensa que ese carácter omni-incluyente abarca también todo lo que "se originará en el futuro de kalpas eternas", entonces allí puede verse el mundo de la armonía preestablecida, que "es un mundo de la autodeterminación del presente absoluto" (XI: 120). Dado que es un mundo de relaciones expresivas que son autonegativas (mundo que va a la desaparición) y autoafirmativas (mundo eterno), "todo lo que existe ha sido determinado desde un pasado eterno y se extiende hacia un futuro eterno" $y$, al mismo tiempo, "todo lo que se manifiesta 'en el futuro de kalpas eternas' es reflejado en el espacio absoluto [y] está incluido en el presente absoluto". En pocas palabras, se trata de un mundo de la armonía preestablecida, se trata de un "mundo del Ser absoluto $\equiv$ Nada absoluta", en el que hay cosas, que se expresa a sí mismo y que es expresado mediante formas (XI: 121).

\subsection{Un mundo histórico}

Como vimos antes, la armonía preestablecida implica que todo existente está incluido en el presente absoluto, y el mundo del presente absoluto es un mundo histórico: "en el mundo histórico todo lo que es, en cuanto realidad individual, debe ser algo que ha sido expresado en Dios, y que ha sido asignado un nombre delante de Dios". La armonía preestablecida implica que "todo lo que se origina históricamente en cuanto autodeterminación del presente absoluto, debe ser algo que ha sido conocido de antemano en Dios". En este sentido, el mundo histórico "tiene dentro de sí mismo un orden, es regulado, [es] un sistema creativo": para expresarlo en sentido lógico Nishida le llama "mundo de la autodeterminación del universal creativo" (XI: 122). Cada individuo expresa al mundo desde su propio punto de vista y la perspectiva del mundo resultante es "proporcional"19 a la perspectiva que resulta del punto de vista de todo otro individuo, de toda otra mónada (XI: 122-123). Por eso, en tanto que el individuo es una singular autodeterminación del mundo, es individuo por oponerse a todo otro individuo y "es individuo por el hecho de expresar al mundo entero [...]: el individuo es único, $[\ldots y]$ debe ser un evento único". Cada individuo, como modo, como punto axial del reflejo del mundo histórico, constituye una relación proporcional: “decir que relaciones 
reflejadas desde puntos de vista totalmente diferentes son idénticas, significará que son mutuamente proporcionales" (XI: 123).

\subsection{Un sistema autofunctorial}

El mundo histórico, en tanto que es un mundo que va de lo hecho a lo que hace, es histórico-corporal, y puede ser visto "enteramente como sistema creativo" en el que "todas estas formas que se determinan a sí mismas histórico-corporalmente son las leyes del mundo histórico" (XI: 123-124), tanto físicas como matemáticas. Todas las mónadas del mundo histórico como sistema creativo "expresan fenómenos idénticos, pero no es necesario que esas expresiones sean idénticas y [...] basta con que sean proporcionales. [... Esto es, basta con que] sus expresiones vengan a ser mutuamente proporcionales". Pero esa proporcionalidad puede pensarse respecto a "cosas' que se expresan a sí mismas de diferentes maneras", esto es, respecto a "cosas' autoperceptivas". Las cosas autoperceptivas se expresan a sí mismas "con carácter de que lo que expresa y lo expresado son uno”, son función de sí mismas y están en relaciones functoriales con todas las demás cosas autoperceptivas. De ahí que Nishida afirme que "el mundo autoexpresivo, que es de por sí y se mueve por sí mismo, en este sentido, es autofunctorialmente autoidéntico" (XI: 124). Por eso, frente a la doctrina aristotélica de la substancia y el sustrato, que es "un dogmatismo metafísico", Nishida afirma que "el mundo que comienza infundamentadamente a partir del hecho de reflejarse a sí mismo, es un mundo en el que el si-mismo viene a ser función de sí mismo ([es] autoidentidad autoperceptiva, es decir, autofunctorial)". En su base, como también en el caso de las leyes físicas, "debe estar la autopercepción intuitivo-activa de nuestro si-mismo en cuanto si-mismo corporal" (XI: 124). Las diversas maneras de expresión son direcciones de lo que se expresa a sí mismo y pueden ser pensadas como múltiples "dimensiones", pero "aunque difiera la dimensión", el mundo histórico que "autoexpresivamente, es decir, autoperceptivamente es uno, es continuo" (XI: 125).

Nishida lleva este pensamiento de una realidad autofunctorial de plenitud activa experienciada a diversas áreas de las ciencias, llama a este punto de vista, "mi positivismo radical" (XI: 124), ${ }^{20}$ y lo liga al operacionalismo de Percy Bridgman ${ }^{21}$ y de Stanley Smith Stevens. ${ }^{22}$ Esta tendencia se describe como: "En general, un concepto significa nada más que un conjunto de operaciones;

20 Cf. James, 1912.

21 VIII: 565; IX: 223, 230, 231-232, 235, 237, 239, 244, 257, 258, 260, 263, 264, 266, 276, 293, 296, 303, 320; Ver el Diario, junio 1 y 11 de1937.

22 IX: 281, 282, 283, 293. 
el concepto es sinónimo con el conjunto de operaciones correspondientes" mediante el cual se determina ese concepto. ${ }^{23}$

\subsection{Un mundo de lo composible}

Un mundo "construido mediante la síntesis de infinitas maneras de ver [...] no podrá ser pensado desde el punto de vista de la lógica abstracta" (XI: 126). Es necesario pensarlo como una unidad de lo múltiple: "todos y cada uno de los individuos debe ser un mundo posible". Ese mundo es la "unidad autonegativa de infinitos individuos" que resulta en un mundo común de aquellos mundos posibles. Por eso, "en cuanto autodeterminación del presente absoluto, este mundo de la realidad actual es un mundo de lo composible" (XI: 125). En el sentido de un constructivismo epistemológico que deriva de la postura de Bridgman y Stevens, Nishida escribe: "podemos decir que este mundo es un mundo construido mediante la síntesis de infinitas maneras de ver, es más, a partir del punto de vista de la manera de ver de [=resultante de diversas] maneras de ver" (XI: 126). Al igual que en el constructivismo epistemológico, Nishida afirma que esa construcción -que epistemológicamente es construcción concreta del mundo composible- resulta impensable "desde el punto de vista de la lógica abstracta, [porque] desde el punto de vista de la lógica abstracta no puede pensarse ni siquiera el mundo de lo composible". Frente a la lógica abstracta, Nishida propone una lógica capaz de aprehender la verdad de hecho, es decir, una lógica que se sustenta en "el punto de vista de la autopercepción de nuestro si-mismo práxico que es intuitivo-activo". La lógica de Nishida es una lógica topológica, una lógica del basho "que toma como principio fundamental al principio de razón suficiente", ${ }^{24}$ en vez del principio de causalidad, y que es capaz de englobar a las demás lógicas como sus momentos (XI: 126).

Nishida se esfuerza por presentar su lógica topológica del basho en términos de la lógica de lo composible bajo la premisa de que el sujeto cognoscente es "un punto creativo del espacio histórico" que está inmerso y se desplaza asintóticamente junto con el movimiento dialéctico del mundo.

Ante todo, el principio de razón suficiente fundamenta el principio de inferencia que deriva de aquel su potencia epistemológica y existencial: "Conocemos un evento mono-ocurrente y único desde el punto de vista de la autopercepción del si-mismo intuitivo-activo". Nuestro si-mismo viene entonces a ser un "aspecto de la autodeterminación del presente absoluto" 
porque el conocimiento originado en la intuición activa implica no sólo la actividad epistemológica sino la actividad creativa de conformar el mundo. Es autopercepción del hacer y de esta manera "conoce al evento mono-ocurrente y único" como un punto que es inicio del mundo en el espacio histórico y que "se constituye tomando como condición a la autonegación de la eternidad" (XI: 126). Cada evento mono-ocurrente y único es enteramente interdependiente con todos los demás eventos mono-ocurrentes y únicos del espacio histórico. En el espacio histórico se da la mutua determinación y dependencia existencial de todo individuo con todos los demás.

Se vislumbra así "un mundo que se constituye mediante la forma de lo composible" (XI: 126-127) en cuanto que es un "mundo de la autodeterminación del presente absoluto", un mundo que en su movimiento dialéctico tiene dos momentos de construcción básicos: a) "de lo hecho a lo activo", en el que a partir de lo hecho se da la "autopercepción de nuestro si-mismo intuitivoactivo"; y b) "de lo activo a lo hecho", en el que el foco creativo mediante su poiesis $\equiv$ praxis va construyendo al mundo composible.

De estos dos movimientos resulta una lógica de lo composible como momento de la lógica del basho de la Nada absoluta. Cuando se refiere al individuo es "la forma lógica de la autopercepción de nuestro si-mismo poietico y debe incluir la intuición activa". Cuando se refiere al mundo, de allí resulta "un mundo que se constituye mediante la forma de la lógica de lo composible, esto es, mediante la forma de la lógica del basho. Podemos decir que allí todo lo que se origina tiene carácter de armonía preestablecida" (XI: 127). En los dos momentos de su movimiento dialéctico, es un mundo del presente absoluto en el que "el futuro eterno es reflejado en el pasado eterno". Por esto también puede decirse que el doble movimiento es "del pasado al futuro" infinitos, y "del futuro al pasado" infinitos. Desde el punto de vista de este doble movimiento también resulta que "el mundo tiene enteramente el carácter de armonía preestablecida” (XI: 128).

\subsection{Mundo del deber infinito}

De ese doble movimiento es sus dos modalidades tenemos, por una parte, que "el pasado es hecho a partir del futuro [y que] el mundo es siempre desde el futuro. El mundo es teleológico. Es más, [...] es un mundo de la libertad" (XI: 128). Pero a su vez, el futuro es construido a partir del pasado, el mundo es siempre desde el pasado: es un mundo predeterminado, es un mundo de la necesariedad. En su filosofía Nishida incluye ambos movimientos: "Ni el punto de partida está en el pasado, ni el punto de llegada está en el futuro; es más, no hay ni siquiera una dirección única, [por lo cual] es un mundo de 
la libertad de la necesariedad y de la necesariedad de la libertad". En otras palabras, dentro de este doble movimiento del mundo histórico, el individuo viene a la existencia desde el pasado y, al mismo tiempo, en tanto que es autodeterminativo tiene libre albedrío: "es reflejado en el pasado eterno y [...] crea un futuro eterno". Pero la necesariedad de que se habló antes "no es una necesariedad material sino que debe ser una necesariedad moral", por lo cual ese mundo histórico dialéctico "es un mundo del deber infinito, es un mundo de la moral" (XI: 128-129).

Leibniz distingue entre necesariedad y deber diciendo que el deber depende del principio de razón suficiente en su mundo de las mónadas que es "un mundo de coexistencia en el que personalidad y personalidad infinitas mutuamente se oponen tomando como centro a Dios". Pero según Nishida, su manera de ver no se libró de una lógica del sujeto, porque en ella "el uno total tiene por entero el carácter de base" de todo y, por tanto, "el todo y el individuo no son verdaderamente autoidentidad de lo contradictorio". Si se considera a Dios como sujeto absoluto, "un Dios así no es el Dios absoluto (ni es la Gottheit [deidad])" sino un Dios relativo "que es lo perfecto frente a lo imperfecto, lo bueno frente a lo malo" (XI: 129). Ese Dios subjetivo (XI: 131) es posteriormente calificado como un "Dios señorial, Dominus" (XI: 400) que es "Señor de los ejércitos" (XI: 396). Es la substancia más elevada pensada como sujeto, pero "no podemos decir que incluya a todo predicado. [...] Desde este punto de vista no resulta la verdadera voluntad libre" y, en consecuencia, "allí no cabe el verdadero individuo, la verdadera personalidad individual" (XI: 129-130). Y si no hay verdadera persona humana, tampoco hay "verdadero mal" y entonces sólo queda afirmar que "el mal es la imperfección" o que el mal es una carencia. Pero "el mundo de la autodeterminación del presente absoluto, por el contrario, debe ser un mundo que incluye a la negación absoluta: debe ser un mundo que incluye [también] al mayor [de todos los] males" (XI: 130).

\subsection{La autodeterminación del presente}

El mundo del presente absoluto que vimos delineado mediante los puntos anteriores, es un mundo de la armonía preestablecida que no se origina ni en el pasado ni en el futuro sino que "es siempre autodeterminación del presente". Una manera de presentarla que frecuentemente utiliza Nishida proviene del pensamiento místico medieval es la "sphaera infinita [...] que no tiene periferia y [en la que] el centro está en todas partes" (XI: 130). Esta idea que, como es sabido, proviene del Liber XXIV philosophorum, Nishida la reencuentra en el pensamiento de Nicolás de Cusa y posteriormente en las Pensées de 
Blaise Pascal. Nos dice Nishida que en la mística medieval "Dios fue pensado como una esfera infinita". Cuando el concepto de la esfera infinita se aplica al mundo del presente absoluto, puede verse que ese mundo incluye también el conflicto: el mundo del presente absoluto como sphaera infinita es "un mundo del conflicto infinito [en el que] individuo e individuo infinitos se oponen, es decir, en el que voluntad y voluntad mutuamente se oponen". Por esto, una vez que Nishida extiende la designación de sphaera infinita al absoluto, al mundo, al individuo, al tiempo, etc., puede verse por qué encuentra que "es semejante [...] al centrum naturae de Boehme" (X: 130). Es un marcador de la centralidad que en referencia al movimiento del mundo histórico designa "el hecho de ser él mismo reflejado", y que en referencia al tiempo dialéctico (tiempo lineal =tiempo circular) apunta a la autodeterminación del presente (XI: 130).

\section{2 ¿Cómo lleva a la filosofía de la religión?}

Mediante los diez puntos anteriores hemos delineado aspectos del mundo de la armonía preestablecida cuyas varias perspectivas son partes interdependientes de la red sistémica de relaciones recíprocas llamada basho cuyo centro temporal es el eterno ahora o presente absoluto. Ahora nos queda por ver cómo a partir de esos planteamientos Nishida trata de configurar una filosofía de la religión.

La primera preocupación de Nishida, ya que en este acercamiento tomó como punto de ingreso la armonía preestablecida de Leibniz, que es un pensador cristiano, es clarificar tres puntos básicos: el Dios cristiano como creador, la relación entre ese Dios cristiano creador y sus creaturas, y el mundo trinitario de vida eterna.

\subsection{Aspectos especificamente cristianos}

\subsubsection{Un mundo creado}

El concepto al que recurre Nishida para clarificar la noción de un Dios cristiano como creador es la "autodeterminación del presente absoluto" (XI: 131). El mundo histórico comienza, como antes se dijo, a partir del hecho de reflejarse a sí mismo. Nishida dice que ese movimiento de autorreflexión es una "determinación sin determinante". Carece de determinante porque es una autodeterminación de la Nada absoluta y, en tanto que es movimiento, la determinación sin determinante apunta a que es un "ir de la Nada al Ser" (XI: 130). Ese movimiento se describe mediante la lógica del basho y cuando se 
introduce la presencia del Dios cristiano, puede decirse que ese ir de la Nada al Ser es "la creación de Dios". Por esta razón dentro del cristianismo puede decirse que tanto el mundo como el individuo son "creata et creans", son al mismo tiempo creados y creadores. Esa identidad no debe verse como una sucesión temporal sino como una "autodeterminación del presente absoluto" (XI: 131), como tiempo concreto en el que la historia viene a ser tal. A este dinámico presente concreto, que implica que "lo dinámico y lo estático son uno" Nishida le llama "movimiento oriental" en el que están implicados creador y creatura (XI: 132). Desde ese punto de vista puede pensarse "el Dios cristiano como creador". Por otra parte, "la relación entre la creatura y el creador" que así se establece, desde la perspectiva de un mundo que se autodetermina, viene a ser "autoidentidad de lo absolutamente contradictorio" (XI: 131).

En ese presente absoluto que es autodeterminación instantánea, la creatura en cada instante se halla "al comienzo y fin del mundo", es decir, se coloca "en un punto de vista escatológico" y encuentra -como hace notar Ranke- que "en el mundo histórico todos los puntos vienen a ser inicio" (XI: 132): en este "mundo del presente absoluto - desde el punto de vista de que el eterno futuro es reflejado en el eterno pasado- todo tiene carácter de armonía preestablecida, todo tiene carácter de destino" (XI: 133). En ese instante eterno, en ese presente absoluto, por una parte, "el mundo es creativo [... y] está henchido de vida" y, por la otra, nuestro si-mismo vive un cuerpo histórico y es "un punto creativo de un mundo así" (XI: 134-135). Por esto Nishida escribe: "Leibniz dice que la mónada es un punto metafísico pero yo pienso que cada uno de nuestros simismos es un punto creativo del mundo creativo" (XI: 135).

Dentro de ese mundo que es creado y creativo, nuestro si-mismo "en cuanto punto de autodeterminación del presente absoluto, se extiende a un futuro eterno y un pasado eterno. Allí donde refleja al pasado eterno, es intelectivo; allí donde refleja al futuro eterno, es volitivo" (XI: 135). Al ser intelectivo, no sólo refleja al pasado eterno sino también expresa al mundo (XI: 143) porque es intuitivo-activo como autoexpresión del mundo. Esto significa que el ser humano comparte con el mundo histórico "el carácter de creata et creans [creado y creador]", por lo cual es capaz de pensar y de llegar a "una verdadera autopercepción". A partir de aquí podemos ver que no sólo es "cogitans" sino que puede intuirse "a sí mismo en cuanto autodeterminación del presente absoluto" y alcanzar la "intuición religiosa" (XI: 144). 


\subsubsection{Un mundo caído}

Nishida clarifica la relación entre el Dios creador y sus creaturas tomando como concepto base la "autoidentidad de lo absolutamente contradictorio" (XI: 131). Para poner el énfasis en la total dependencia existencial de ese Dios, afirma que "el mundo comienza del pecado [... y que] la existencia de nuestro si-mismo está en el pecado". Sin embargo, "como siervos de Dios tenemos vida eterna" en un mundo de libertad infinita (X: 130). De allí que nuestro si-mismo pueda ver y reflejar el mundo histórico (es cognitivo) y, al mismo tiempo, en "un mundo de la voluntad, un mundo del esfuerzo" actuar en ese mundo y formarlo (es volitivo). En la unión de conocimiento y voluntad comienza el si-mismo que se constituye allí donde constituye al mundo; al mismo tiempo, "donde se constituye el si-mismo, allí se constituye el mundo" (XI: 133).

\subsubsection{Un mundo de vida eterna}

Otro aspecto que es específicamente cristiano, es el mundo trinitario. ${ }^{25}$ Nishida dice que de la verdadera autopercepción del ser humano en una religión surge el requerimiento de vida eterna (XI: 133). En este sentido, donde existimos y actuamos como siervos de Dios, allí "tenemos vida eterna" (XI: 130). Nishida afirma que ese "mundo de la vida eterna -si se utiliza una expresión cristiana- es en su base un mundo de la Trinidad con carácter de autoidentidad de lo absolutamente contradictorio: Padre, Hijo y Espíritu Santo". O bien, sin recurrir necesariamente a conceptos cristianos, también puede decirse que "todo lo que es, es órgano de la vida eterna" (XI: 333). ${ }^{26}$

\subsection{Hacia una filosofía de la religión}

Una vez que Nishida en alguna medida toca aquellos tres puntos específicamente cristianos y señala una manera de abordarlos que es congruente con su visión filosófica, puede avanzar en su intento de retomar la "armonía preestablecida" de Leibniz como concepto guía para la elaboración de una filosofía de la religión. Para ello enfatiza los temas comunes a las varias religiones, especialmente al budismo y al cristianismo. Aborda cuatro temas principales desde su propia interpretación de la armonía preestablecida: la religión como problema del si-mismo, la autopercepción, la libertad infinita, y la moralidad. 


\subsubsection{La religión como problema del si-mismo}

Una condición indispensable para que nuestro si-mismo entre a la religión es la percepción de la autoidentidad de lo contradictorio en el fondo de la propia existencia. En un nivel inicial llegamos a ver que en el mundo por doquier está presente la contradicción y vemos también el antes mencionado "movimiento oriental" nishidiano que es autoidentidad de los contradictorios. Esta autoidentidad implica una doble perspectiva: a) Cuando con carácter de autoidentidad de lo contradictorio el mundo se determina a sí mismo y esa determinación es una autodeterminación del presente absoluto, entonces nuestro si-mismo se conoce a sí mismo, se percibe a sí mismo. b) A la inversa, "allí donde nuestro si-mismo se autopercibe, allí el mundo se determina a sí mismo". De esta manera puede verse un caso paradigmático de la autoidentidad de lo contradictorio, a partir del cual puede decirse que "La lógica de la autopercepción es la lógica de la constitución del mundo, y la lógica de la constitución del mundo es la lógica de la autopercepción" (XI: 139).

En un nivel un poco más profundo llegamos a darnos cuenta de que en ese movimiento oriental el instinto y la emoción, el intelecto y la voluntad, el cuerpo y la mente aunque son diversos forman una unidad (XI: 134). Encontramos entonces que nuestro si-mismo individual es "un elemento formativo del mundo histórico [que] está actuando mediante la lógica de la autopercepción" (XI: 139). Formamos al mundo "mediante el hecho de que el si-mismo refleje al mundo" (XI: 140). En ese nivel podemos también llegar a darnos cuenta de que la ciencia y la moral se originan a partir de esa unidad. Pero esa unidad ya es una autoidentidad de lo contradictorio (XI: 134). Esto se debe a que llegamos a convencernos -equivocadamente- de que por nuestro propio poder nos cambiamos a nosotros mismos y formamos el mundo.

Aquí se presenta la oportunidad para alcanzar una todavía mayor profundización de nuestra autopercepción, ya que tener el convencimiento de ese poder nos acarrea angustias y penalidades. Encontramos que la existencia de nuestro si-mismo es profundamente autocontradictoria y que "esta contradicción no puede ser eliminada con las fuerzas del si-mismo [y tampoco] con la acción de la fuerza moral". La ciencia no nos libra de la autocontradictoriedad de nuestra vida y al recurrir a la acción moral nos damos cuenta de que ésta "se fundamenta en la autoidentidad de lo contradictorio" (XI: 140).

Aunque no es el único camino, al ir profundizando nuestra autopercepción y llegar hasta el nadir, hasta las profundidades del abismo, por primera vez nos encontramos con la contradicción absoluta. En cada uno de los múltiples niveles nos vamos tornando cada vez más individuales, y cuando el nivel 
más profundo se desborda, llegamos a percibirnos desde "el punto de vista de la confrontación con el absoluto en cada instante" (XI: 132). Pero, una vez agotados los recursos individuales y colectivos del ser humano, queda al descubierto "la raíz del requerimiento religioso del hombre" (XI: 140). Alcanzamos entonces a vislumbrar que, desde nosotros, hay una distancia insalvable entre hombre y Dios: "Absolutamente no hay camino del hombre a Dios" (XI: 131).

Esta manera de plantear el problema "no toma psicológicamente como punto de partida el cogito de nuestro si-mismo como Descartes", y Nishida encuentra que en la filosofía occidental la "docta ignorantia, de Nicolás de Cusa es lo que más se acerca a esto" (XI: 138). Para plantear el problema Nishida toma como punto de partida el hecho de que llegar a vivenciar la autoidentidad de lo contradictorio es autopercepción. Es decir, "Cuando nuestro si-mismo en el fondo de nuestra autopercepción llega a percibir esta autoidentidad de lo contradictorio, entonces es religioso" (XI: 134). Nos dice que la verdadera autopercepción es, citando a Suzuki Daisetz, "la discriminación de la no-discriminación". Esto implica, en un sentido lógico como dice el Sutra Prajñaparamita, que "todos los corazones son no-corazón por eso se llama corazón" (XI: 138). Por otra parte, Nishida afirma que "cuanto más individuales nos tornamos, tanto más nos acercamos a Dios" (XI: 131).

Además del despertar de la consciencia de la autoidentidad de lo contradictorio y de la vivencia autoperceptiva, nuestro si-mismo llega a darse cuenta de que su religiosidad no es obra propia: "cuando se ha llegado a penetrar verdaderamente el si-mismo entonces [encontramos] que eso era el llamado de Dios. A partir de allí, tanto la ciencia como la moral son religiosas; es más, vestir, beber y comer deben ser, todos, [eventos] religiosos. La religión es el problema del si-mismo" (XI: 134). A partir de aquí podemos ver otros aspectos que son igualmente importantes para una filosofía de la religión.

\subsubsection{Un mundo de la autopercepción}

Para hacer un poco más comprensible su posición, frente a quienes piensan "la autopercepción a partir del conocimiento o de la voluntad" o piensan un desarrollo a partir del inconsciente, Nishida apunta que en el primer caso se trata de quienes piensan lo concreto sobre la base de lo abstracto, y que en el segundo se trata de quienes piensan lo manifiesto a partir de lo latente, y señala que "del simple inconsciente no resulta la consciencia". En este segundo caso, la gente piensa con lógica objetual y asume que "lo que todavía no se manifestaba se viene a manifestar en el tiempo" (XI: 135). 
La autopercepción de que habla Nishida no es un simple proceso temporal, ni comienza de las facultades o del inconsciente del individuo sino de la situación existencial del si-mismo. Explica con un ejemplo: "en sentido psicológico, la autopercepción del niño [comienza] a partir de [su] oponerse a la gente de su alrededor" (XI: 135). Más explícitamente, nos dice que nuestra autopercepción "comienza a partir del hecho de que el presente se determine a sí mismo" (XI: 136). En este sentido se trata de un proceso temporal, pero no como un proceso teleológico porque entonces resultaría que el final del proceso estaría allí desde el comienzo. Por el contrario, "el proceso temporal [...] se constituye como autodeterminación del basho" y allí la autopercepción tiene carácter de "autodeterminación del presente absoluto" (XI: 135-136).

El movimiento circular de reflejarse a sí mismo dentro de sí, es la forma general de la autodeterminación. Esa forma implica tiempo circular y todo lo que en ella se manifiesta es activo: implica el entrejuego de lo latente y lo manifiesto. Cuando hablamos de la autodeterminación del mundo ya ahí está implicada la formación del mundo objetivo. Ahora bien, "la forma de la autopercepción es la forma misma [en que] el presente se determina a sí mismo" (XI: 136). En este sentido, la autopercepción de nuestro si-mismo es, en el aspecto noemático, hacer consciencia del si-mismo, pero en el aspecto noético, es retornar al origen del si-mismo, es decir, es "entrar al origen de la constitución del mundo" (XI: 137). Por eso "la autopercepción de nuestro si-mismo es ya la autoexpresión del mundo; y la autoexpresión del mundo es ya la autopercepción de nuestro si-mismo" (XI: 133). En otras palabras, la autopercepción del si-mismo "no es penetrar subjetivamente a lo interior sino, más bien, enteramente salir a lo externo [...] Y todavía más, es que el dentro se torne fuera y el fuera se torne dentro" (XI: 136-137). Por eso la autopercepción del si-mismo es "consciencia de la existencia del si-mismo" y, ante todo, es "autopercepción activa" (XI; 137): "autopercibirse es que el cognoscente sea lo conocido, que lo pensante sea lo pensado. En sentido amplio, es que lo que es expresado sea lo que expresa" (XI: 137-138).

Desde esta manera de entender la autopercepción, Nishida afirma que la "docta ignorantia" de Nicolás de Cusa, liberándose de la lógica del sujeto, "debe ser una lógica de la autopercepción" y que su "coincidentia oppositorum [...] podrá ser expresada muy bien mediante la lógica del basho" (XI: 138-139). Por una parte, esto implica apartarse de la lógica del sujeto que ha sido parte de la construcción doctrinal del cristianismo, pero también requiere apartarse de la lógica del objeto que reifica todo lo que es individual. Nishida dice que su lógica de la autopercepción debe entenderse estrictamente en el sentido de que "El hecho de que el individuo sea enteramente individuo es que el todo 
sea todo; el hecho de que el todo sea enteramente el todo, es que el individuo sea individuo". En la lógica del basho, "cuanto más y más profundamente reflexionamos en el fondo del si-mismo acerca de la base de nuestro si-mismo, tanto más nos oponemos externamente al Dios absoluto". Por no ser lógica del objeto, no es panteísmo, y por no ser lógica del sujeto, no es misticismo. En la lógica de la autopercepción, "nuestro si-mismo es, cognitiva y activamente, un infinito proceso que tiene carácter de autoidentidad de lo contradictorio" y, desde este punto de vista "la lógica de la autopercepción es el axioma de nuestra vida histórica” (XI: 139).

\subsubsection{Un mundo de la libertad infinita}

Si se piensa la autodeterminación del presente absoluto con lógica objetual, de allí resultaría un panteísmo. Por el contrario, si se le piensa con la lógica del basho, la autodeterminación del individuo establece una perspectiva del mundo en la que una persona única individual "se opone a la persona única de Dios como unidad total". Por eso desde este punto de vista se puede hablar de las relaciones entre seres humanos y Dios, o en lenguaje cristiano, de "las relaciones entre creador y creatura" (XI: 132). Pero como individuo único que es autodeterminación del presente absoluto - en tanto decir que "[ir] desde el pasado eterno es [venir] desde el futuro eterno"- nuestro si-mismo "es enteramente volitivo", existe en un mundo de libertad infinita y está en relación de libertad infinita frente a Dios. En esa relación está en juego la vida eterna: "quienes desobedecen a Dios mueren, quienes retornan a Dios entran a la vida eterna" (XI: 133).

\subsubsection{Un mundo moral}

Si se considera al ser humano en el tiempo lineal, en el que sólo se ve que "va del pasado al futuro, [...] el ser humano es material" y si, además, sólo se ve que "va de lo que es reflejado a lo que refleja, es instintivo, es animal" (XI: 130). Se trata de una visión unilateral que debe ser complementada con la perspectiva del tiempo circular en la que el ser humano "como autodeterminación del presente absoluto [...] tiene anhelos e intereses propios" (XI: 131). En la imbricación de esas perspectivas, "tanto el conocimiento como la voluntad [se originan] a partir de la autopercepción de nuestro si-mismo" (XI: 134). Cuando la perspectiva individual se toma como punto de partida de la reflexión, el si-mismo busca hacer todo con su propia voluntad, está lleno de "poder de sí" y quiere hacer todo por sus propias fuerzas (XI: 131). En esa perspectiva unilateral, viene a ser un individuo único, cognitivo y volitivo "en un mundo en el que el presente se determina a sí mismo" (XI: 134). El 
resultado es que si sólo se le considera como racional o como desiderativo, no puede ser verdaderamente moral. Sólo cuando se coloca en un mundo de libertad infinita, por ser un individuo único puede ser desleal, o bien, "en cuanto que es creado y crea, es divinamente moral". Entonces queda en claro que ese individuo único no es divino por ser moral "sino que es moral debido a que es divino" (XI: 131). En un mundo que "comienza a partir del hecho de reflejarse a sí mismo. Tanto la moral como la cultura se fundamentan religiosamente" (XI: 145-146).

Cuando con lógica objetual se examina la religión como problema del conocimiento, o cuando mediante el requerimiento moral se investiga la religión, todavía no se abandona el punto de vista que ve todo desde la "autopercepción de nuestro si-mismo" (XI: 134). Aquí todavía el punto de vista no es suficientemente profundo. Sólo cuando arrancando del mundo histórico -que "es autoformativamente creativo, infinitamente procesual y temporal", y cuya forma se determina a sí misma-, se llega a ver que "nuestro si-mismo en cuanto elemento creativo de un mundo creativo toca a la autoexpresión del mundo infinito". Allí se puede acceder al punto de vista de la autopercepción religiosa y ese elemento creativo se encuentra entonces en un mundo que es "enteramente moral" (XI: 145).

\section{Elementos para una filosofía de la religión}

Una vez que hemos visto la manera en que Nishida examina a su manera la armonía preestablecida de Leibniz, podemos señalar cómo esto le permite avanzar hacia una filosofía de la religión. Se trata de cuatro puntos principales: la manera de ver la Deidad cristiana y el Vacío del budismo, lo que es la religión, la entrada a la fe, y algunos apuntamientos para una filosofía de la religión.

\subsection{La Deidad y el Vacío del Prajñaparamita del budismo}

Los pensadores cristianos consideran el budismo como panteísta, pero Nishida dice que aunque en el cristianismo predomina un Dios pensado con lógica del sujeto, que es un Dios subjetivo, en tanto que también hay un pensamiento como el de la Deidad (XI: 129), el cristianismo no se libra de un panteísmo del que tampoco Leibniz escapa (XI: 131). Señala una unilateralidad inmanentista y cuestiona si "negando estas relaciones, ¿[debemos] pensar a Dios absolutamente como trascendente?" Pero lo que de allí resultaría sería "un Dios simplemente negativo, como un Dios lejano que, por el contrario, no se libraría de ser relativo. Un Dios escondido, Deus absconditus, no puede ser 
llamado Dios absoluto". Por eso Nishida dice que "el verdadero Dios es [...] más bien, en occidente, la Deidad (Gottheit), como le llaman los místicos: es el Vacío del Prajñaparamita" (XI: 131). Por otra parte, Nishida afirma que incluso en la teología dialéctica cristiana subsiste el punto de vista de la lógica objetual y, por eso, desde ese punto de vista no se llega a pensar que "el Dios verdaderamente absoluto debe ser algo que por entero nos trasciende y, al mismo tiempo, nos envuelve" (XI: 131). Cuando sólo queda un Dios subjetivo trascendente se pierde el mundo objetivo y "allí donde el mundo objetivo no viene a ser el trasfondo, no hay ni penalidades, ni pecado, ni salvación. Allí no se constituye ninguna religión" (XI: 142).

Aunque pudiera decirse que en comparación con el cristianismo el budismo es ahistórico, en realidad en ambos casos se trata del "mundo del presente absoluto [que] es enteramente temporal-espacial y espacial-temporal", y sólo hay diferencia de énfasis sobre lo espacial o lo temporal. En el cristianismo el mundo es visto privilegiando lo espacial sobre lo temporal y en el budismo se privilegia lo temporal sobre lo espacial al ver un "mundo de la originación y desaparición" continuas (XI: 142). La razón es que en el cristianismo "este mundo es un mundo de la creación de Dios" mientras que en el budismo el mundo es visto desde una dimensión histórica como "mundo del karma". Mientras en el cristianismo se tiende a poner énfasis en el Dios creador de todo, en el budismo se enfatiza un mundo impermanente, "un mundo de la originación y desaparición infinitas, [que] es simplemente un mundo de penalidades". Sin embargo, en ambos casos, desde el hombre "absolutamente no hay camino de liberación" (XI: 142).

Nishida hace referencia al budismo Zen y al budismo de la Tierra Pura. En el budismo Zen, cuando el esfuerzo queda sólo en la voluntad humana, cuando forzando nuestro cuerpo lo arrastramos para dar testimonio de los diez mil seres, allí sólo hay obnubilación: "comprobar los diez mil dharmas arrastrando al si-mismo, es descarrío". En cambio, cuando mediante una intuición (XI: 141) logramos penetrar hasta el origen de la autopercepción eso es ya un satori, una iluminación. No se trata de un misticismo sino de "un ver que no es ver objetualmente cosa alguna, [sino que] es ver sin ver" (XI: 143), es que el si-mismo sea atestiguado "por los diez mil dharmas" (XI: 141), como dice el Shôbôgenzô de Dôgen. ${ }^{27}$

En el budismo de la Tierra Pura, los seres humanos pueden liberarse de la obnubilación en este mundo de penalidades eternas sólo "mediante la compasión 
del Buda" dado que mediante el Voto del Buda "se nace en la Tierra Pura". Esta vivencia se alcanza "mediante el hecho de creer en el Buda y tomar refugio en él" (XI: 142). En tanto que se requiere un cambio en la orientación vital, una metanoia, y un absoluto abandono y entrega de sí, "hay puntos de contacto [...] entre la secta de la Tierra Pura y el cristianismo". Nishida nos dice que "en ambos casos, la esencia de la religión reside en penetrar hasta el verdadero origen de nuestro si-mismo en cuanto autodeterminación del presente absoluto". Sin embargo, cuando se ven estas dos religiones desde otra perspectiva, "la una trasciende este mundo en dirección de lo trascendente, en dirección objetiva, mientras que la otra lo trasciende en dirección de lo inmanente, en dirección subjetiva" (XI: 143).

\subsection{Religión}

La religión, ante todo, "debe ser [un] requerimiento de vida eterna, es decir, debe ser un requerimiento de la verdadera autopercepción del hombre" (XI: 133). Básicamente, no es un ejercicio cognitivo con lógica objetual, ni un requerimiento moral en la voluntad. Aunque "nuestro si-mismo es cognitivo y volitivo, [y] puede decirse que el si-mismo comienza allí donde conocimiento y voluntad se unen", este no es el aspecto más básico de nuestro si-mismo ya que "tanto el conocimiento como la voluntad se originan de la autopercepción de nuestro si-mismo, [esto es,] en cuanto existencia que tiene carácter de autoidentidad de lo contradictorio". Por esto puede decirse que "cuando nuestro si-mismo en el fondo de nuestra autopercepción llega a percibir esta autoidentidad de lo contradictorio, entonces es religioso". La vivencia de la contradicción absoluta es un camino para entrar a la religión. Esta vivencia parecería ser totalmente una moción desde el ser humano, pero habrá que tener en cuenta que "cuando se ha llegado a penetrar la verdad del si-mismo entonces [encontramos] que eso era el llamado de Dios" (XI: 134).

Nishida hace notar que la religión: a) no es "pensar a Dios como el principio más elevado desde el punto de vista del conocimiento"; b) no es "colocar la existencia de Dios como requisito desde el punto de vista de la moral"; c) no es algo que "simplemente se fundamente en una vivencia mística subjetiva" (XI: 137); d) no es una práctica diseñada "simplemente para tranquilidad individual" (XI: 144); e) ni es "algo que esté completamente desligado de la sociedad, las instituciones o la cultura" (XI: 145).

Lo anterior permite llegar a ver que "el punto de vista de la religión es el punto de vista de la autopercepción" (XI: 137). Por esto entrar a la religión es básicamente "el problema del si-mismo" (XI: 134). Como dice Takemura Makio, "el problema de la religión está en qué existencia sea el si-mismo y 
dónde se coloca". ${ }^{28}$ Además, en la base de nuestra autopercepción "siempre está la autoidentidad de lo contradictorio" (XI: 140) y ese punto de vista de la consciencia autoperceptiva es "base del conocimiento y de la moral" (XI: 137) y sobre esa base, todos los actos de la vida diaria son eventos religiosos (XI: 134), como antes se dijo.

\subsection{La entrada a la religión}

La entrada a la religión se caracteriza por no ser producto de los designios del ser humano sino por una moción o vocación que proviene del Absoluto. Al penetrar de lleno en la autoidentidad de lo contradictorio adviene la autopercepción y conforme esta se profundiza nos encaminamos hacia un cambio de vida, que es el arrepentimiento religioso, es decir, nos orientamos hacia una metanoia. En el fondo de nuestra vida experimentamos la frase agustiniana, "nuestro corazón no tiene sosiego hasta que descanse en ti" (Agustín de Hipona. Confesiones [I, 1]) y finalmente podemos vivenciar que ese movimiento mismo y entrar a la fe es ya "el llamado de Dios". Por eso Lutero en el Prefacio a la Epístola a los Romanos [I, 16] escribe que "la fe es obra de Dios que opera en nosotros, nos transforma y nos hace renacer de Dios, da muerte al viejo Adán, nos hace hombres completamente diferentes, $\mathrm{y}$ trae consigo al Espíritu Santo" (XI: 140-141).

A esto se le puede llamar fe o se le puede llamar satori, iluminación (XI: 140). Allí llegamos a percibir que "el mundo de la autodeterminación del presente absoluto [en el que] en cuanto autoidentidad de lo contradictorio el presente se determina a sí mismo, es religioso en la base de su constitución". El mundo que se constituye religiosamente es un mundo en el que "la multiplicidad individual y la unidad total se oponen con carácter de autoidentidad de lo contradictorio" (XI: 141) y es enteramente algo que se va formando en la historia $\mathrm{y}$, a su vez, va formando a la historia. Nuestro si-mismo tiene también ese mismo carácter. Por eso puede decirse que "este mundo mismo [es] autodeterminación del presente absoluto" y cada uno de nosotros los seres humanos que lo habitamos reflejamos ese mundo y somos su autodeterminación: "nuestro si-mismo no es sino la autodeterminación instantánea del mundo [en el que] el presente se determina a sí mismo. Aquí el mundo es enteramente religioso" (XI: 145).

Quienquiera que se ha autopercibido religiosamente en este sentido y ha entrado a la fe religiosa, "viene a ser "señor del lugar donde se encuentre", 
(como reza la expresión Zen). Se torna completamente activo, "su conocer es docta ignorantia, es discriminación de la no-discriminación", se despoja de su yo y su egocentrismo, y "desde el fondo de este no-yo se desborda una compasión infinita” (XI: 144).

\subsection{Hacia una filosofía de la religión}

Nishida encuentra que hay dos maneras de ver la religión en la filosofía occidental consonantes con su esfuerzo para construir una filosofía de la religión: una es la de Nicolás de Cusa y la otra es la de Leibniz. La razón es que Nicolás de Cusa por una parte "se ve llevado a la teología negativa", pero por otra "quería ser lógico". Es un precursor del esfuerzo de Leibniz, que era también matemático. Su pensamiento matemático puede verse, por ejemplo, cuando "dice que toda investigación es una comparación que depende de la proporcionalidad con lo ya conocido (comparativa est omnis inquisitio)" (XI: 138). En esa indagación, "lo más grande debe ser lo que ha acabado con la oposición comparativa, debe ser algo mayor que el cual no hay nada", y ese uno que ha trascendido toda oposición comparativa es lo máximo que se une a lo mínimo. Desde esta perspectiva comparativa de proporcionalidad matemática, para Leibniz, "ese es Dios". Igual que otros pensadores, también Leibniz utiliza la imagen de la esfera infinita para expresar a Dios. En la teología positiva se dice que Dios es origen de todo, mientras que en la teología negativa no debe nombrarse. Por esto, Dios “es Ser absoluto y, al mismo tiempo, es Nada absoluta" (XI: 138). Sin embargo, podemos reconocer una oposición entre Dios y el ser humano en el mundo: "cuanto más personalmente individual se torna nuestro simismo, tanto más se enfrenta al Dios único y absoluto" en este mundo histórico que viene a tener como base de su constitución "lo religioso con carácter de trascendente $\equiv$ inmanente, e inmanente $\equiv$ trascendente". Nishida escribió que a partir de estas relaciones trilaterales (Dios-hombre-mundo) pensaba "organizar una filosofía de la religión" (XI: 141).

En el mundo histórico hay diversidad de religiones que se originan "mediante las formas de autoidentidad de lo contradictorio: Dios y seres humanos" (XI: 141). Pero las diversas formas determinadas resultantes "deben interpenetrarse" (XI: 142). Nishida dice que "los diversos problemas de una filosofía religiosa deben pensarse desde este punto de vista" (XI: 141). Por eso su filosofia de la religión toma como referentes los puntos de semejanza y diferencia entre 
algunas de las diversas religiones, en especial el cristianismo y el budismo como religiones universales. ${ }^{29}$

\section{Conclusión}

En este escrito es posible atisbar la manera en que Nishida va construyendo su filosofía de la religión. Podemos ver cómo poco a poco va construyendo las diferentes áreas de su filosofía en su confrontación con obras de los antiguos, en el diálogo con los escritos de sus contemporáneos, y mediante su propia reflexión y reconstrucción.

Nishida señala una diferencia respecto a Leibniz. Nos dice que en el caso de Leibniz la armonía preestablecida fue una hipótesis, mientras que Nishida la toma como "un principio lógico fundamental de la construcción del mundo histórico" (XI: 116).

Podría servir esta presentación para alejar de nuestra mente la idea de que Nishida, con una serie de presuposiciones provenientes del budismo (en especial del budismo Zen y de la secta de la Tierra Pura), simplemente fue encontrando doctrinas filosóficas y teológicas a rebatir en la filosofía occidental y en el cristianismo. Más que de una contrastación de doctrinas, se trata de una reflexión basada en su propia vivencia, que en este caso viene a ser el referente último para la construcción de su filosofía de la religión.

Por otra parte, hemos podido ver cómo en su filosofía de la religión se va perfilando el diálogo entre budismo y cristianismo en tres aspectos: a) el contraste entre el substancialismo subyacente a la idea cristiana de creación y la Nada absoluta ( $m u$ ) del budismo; b) la entrada a la religión y posible semejanza entre metanoia y satori; y c) algunos puntos de contraste entre el ágape cristiano y la compasión (jihi; maha-karuna) budista.

\section{Bibliografía}

BRIDGMAN, P. W. "The Nature of Physical Theory”. New York: MacMillan, 1927. Accesible en: www.marxists.org (Consultado el 2 de mayo, 2014).

COUTURAT, L. "La logique de Leibniz”. Paris: Félix Alcan, Éditeur, 1901. [Online] Accesible en: http://archive.org (Consultado el 4 de agosto 2013).

JACINTO, A. Z. "The Philosophy of Religion in Nishida Kitarô: 1901-1914". Théologiques, 20, No. 1-2. Montréal: Université de Montréal, 2012. Les philosophes de l'École de Kyôto et la théologie, pp. 39-60. 
JAMES, W. "Essays in Radical Empiricism". New York: Longmans, Green, and Co., 1912. Accesible en: http://archive.org (Consultado el 19 de abril, 2014).

JANET, P. “Oeuvres philosophiques de Leibniz”. Paris: Félix Alcan, Éditeur, 1900. Accesible en: http://gallica.bnf.fr (Consultado el 5 de febrero, 2014).

KOASAHI, S. "Leibniz no monado to chikara- Nishida to Lenin" (La mónada de Leibniz y la fuerza- Nishida y Lenin). En: Shizen benshôhô (Dialektik der Natur) no tame ni (Para una dialéctica de la naturaleza). Marzo 2013. Accesible en: www. dialektik21.web.fc2.com/leibniz.html (Consultado el 10 de abril, 2014).

KURUSU, T. "Einfluss der Monadenlehre von G. W. Leibniz auf die Philosophie von Kitarô Nishida?" Tesis de Maestría-Doctorado. Munich: München Universität, 2005. Parcialmente accesible en: www.bvbr.bib-bvb.de (Consultado el 12 de abril, 2014).

LEIBNIZ, G.-W. "Discours de Métaphysique". H. Lestienne Ed. Paris: Félix Alcan, Editeur, 1907. Accesible en: http://gallica.bnf.fr (Consultado el 8 de abril, 2014).

"The Monadology and other Philosophical Works". Oxford: Oxford University Press, 1925 (1898). Accesible en: http://books.google.com (Consultado el 8 de abril, 2014).

MASUYAMA, H. "Kanto hihan tetsugaku no zenshi: 18-seiki doitsu ni okeru Leibniz juyô to Hume juyô" (El siglo anterior a la filosofía crítica de Kant: La recepción de Leibniz y Hume en la Alemania del siglo XVIII). En: Hokkaido University. Research Journal of Graduate Students of Letters, 13, pp. 42-61, 2013. Accesible en: http://hdl. handle.net/2115/54062 (Consultado el 8 de abril. 2014).

NISHIDA, K. "Nishida Kitarô Zenshû" (Obras completas de Nishida Kitarô). Tokio: Iwanami shoten. 1965-1966. 19 Vols.

ODIN, S. "Kankyô riron toshite no Whitehead no perspective-shugi - Nihon-teki shizen no gainen ni taisuru process shisô" (El perspectivismo de Whitehead como teoría del ambiente- la idea de proceso vs. El concepto japonés de naturaleza). En: Sophia University. Jôchi Tetsugaku-shi, 24, pp. 71-88, 2012. Accesible en: http:// repository.cc.sophia.ac.jp/dspace/handle/123456789/34673 (Consultado el 11 de abril, 2014).

ÔKUBO, D. D. (ed.). "Shôbôgenzô". Tokio: Chikuma shobô, 1971.

ÔNISHI, M. "Honshitsu no kagami to kami no kagami (zenpan). Nishida ni miru Hegel to Leibniz" (Mirror of Essence and Mirror of God (1/2)- Hegel and Leibniz in the philosophy of Nishida). En: Ritsumeikan University. Ritsumeikan Ningen-gaku kenkyû. 5, pp. 121-130, 2003. Accesible en: http://ci.nii.ac.jp/naid/110006375202 (Consultado el 11 de abril, 2014).

. "Leibniz to bukkyô to Nishida: mado no aru monadoroji- (Monadologie mit offenem Fenster). Ritsumeikan University. The Ritsumeikan Literature Review. 625, pp. 1014-1026, 2012. [Online] Accesible en: http://ci.nii.ac.jp/vol_issue/nels/ AN00289973/ISS000483246_en.html (Consultado el 11 de abril, 2014).

. "Leibniz to Nishida- fukuzatsu-kei wo baikai ni shite" (Leibniz und Nishidain Vermittlung von der Komplexsystem-theorie). En: Nihon Leibniz Kyôkai. Studia 
Leibnitiana Japonica.. Leibniz kenkyû, 1, pp. 1014-1026, 2010. [Online] Accesible en: http://www-cc.gakushuin.ac.jp (Consultado el 11 de abril, 2014).

RUSSELL, B. "A Critical Exposition of the Philosophy of Leibniz". Cambridge: University Press, 1900. [Online] Accesible en: http://archive.org (Consultado el 17 de marzo, 2014).

SAITÔ, J. "Nishida Tetsugaku to san'ichi-ronteki sôzô-ron. The Philosophy of Nishida Kitaro and the Theology of Trinitarian Creation". En: Dôshisha University. Kirisutokyô kenkyûu (Studies in Christianity). 71, Nr. 2, pp. 95-115, 2009. [Online] Accesible en: http://doors.doshisha.ac.jp/webopac/../bodyid=TB12245713 (Consultado el 13 de abril, 2014).

SAKAI, K. "G. W. Leibniz und Kitarô Nishida. Die Frage nach dem Selbst", Studia Leibniziana, 40, No. 1, pp. 92-113, 2008. [Online] Accesible en: http://philpapers.org/ rec/SAKGWL (Consultado el 13 de abril, 2014).

SHIMIZU, T. "Serres, sôzô no monado- Leibniz kara Nishida made" (Serres, la mónada creativa - de Leibniz a Nishida). Kioto: Tôkyû-sha, 2004.

SPINOZA, B. "L'Éthique". Paris: Gallimard, 1970.

TAKEMURA, M. "Nishida Kitarô to Bukkyô. Zen to Shinshû no kontei wo kiwameru" (Nishida Kitarô y el Budismo: investigar el fondo del Zen y de la secta Shin). Tokio: Daitô shuppansha, 2002.

TARDE, G. "Monadologie et sociologie". En: Tarde, Gabriel. Essais et mélanges sociologiqes. Lyon-Paris: Editions Storck et Masson, 1895. Ed. Institut Synthélabo, 1999.

YAMAMOTO, A. "Seishin byôrigaku kara mita Nishida Kitarô bannen no jigaron- Jiheishô, bunretsubyô-ron no kiso-zuke no tame ni" (Ich-Struktur nach Nishida interpretiert von Husserlscher Psychopathologie I: Fundierung der fruhkindlichen Autismus- und der Schizophrenie-Theorie). Osaka: Osaka Kyôiku Daigaku. Ôsaka Kyôiku Daigaku kiyô, IV Parte, 46, No. 2, pp. 323-336, Enero 1998. [Online] Accesible en: http://ci.nii.ac.jp/11000119357 (Consultado el 8 de abril, 2014).

YONEYAMA, M. "Jôhô-gaku no kiso" (Fundamentos de la informática). Yokohama: Ômura shoten, 2002.

"Monadoloji- no bigaku: Esthétique de monadologie. Leibniz/Nishida Kitarô/Alain”. Nagoya: Nagoya Daigaku Shuppan-kai, 1999. 